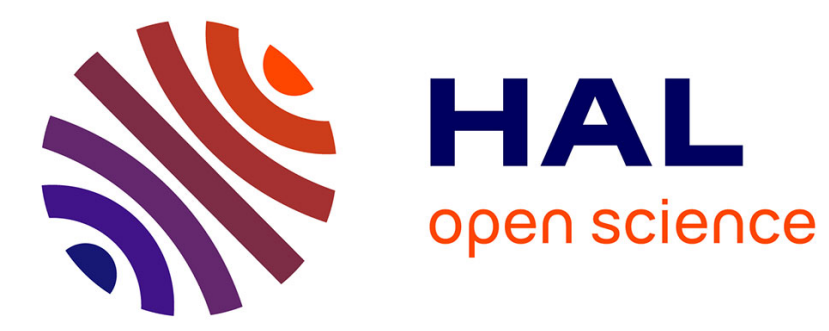

\title{
Dynamiques de classe et efficacité scolaire
}

Sophie Genelot, Frédéric Tupin

\section{To cite this version:}

Sophie Genelot, Frédéric Tupin. Dynamiques de classe et efficacité scolaire. Les Dossiers des sciences de l'éducation , 2003, 10, pp.109-130. halshs-00004890

\section{HAL Id: halshs-00004890 \\ https://shs.hal.science/halshs-00004890}

Submitted on 7 Jun 2021

HAL is a multi-disciplinary open access archive for the deposit and dissemination of scientific research documents, whether they are published or not. The documents may come from teaching and research institutions in France or abroad, or from public or private research centers.
L'archive ouverte pluridisciplinaire HAL, est destinée au dépôt et à la diffusion de documents scientifiques de niveau recherche, publiés ou non, émanant des établissements d'enseignement et de recherche français ou étrangers, des laboratoires publics ou privés.

\section{(이) $\$$}

Distributed under a Creative Commons Attribution - NonCommercial - NoDerivatives| 4.0 


\title{
Sophie Genelot
}

Université des Antilles et de la Guyane / IREDU-CNRS

Frédéric Tupin

Université de la Réunion / LCF UMR 8143 CNRS

\author{
Avec la collaboration de Sandrine Aeby \\ et Jean-François de Pietro,
}

IRDP, Neuchâtel`

\section{Dynamiques de classe}

\section{et efficacité scolaire}

\section{Résumé}

La recherche présentée ici a pour objet l'efficacité des pratiques enseignantes envisagée dans la dynamique des processus d'enseignement-apprentissage. L'objectif central poursuivi consiste à mettre en relation des observations de classes et les acquisitions des élèves concernés afin d'être mieux à même d'expliquer sous l'effet de quels vecteurs se construisent les connaissances/compétences des élèves. En arrière plan, au niveau théorique, cela implique, de fait, de conjuguer paradigme « processus-produit » et paradigme « écologique »

Les résultats tendent à montrer que la mise en commun des démarches d'analyse des pratiques et des démarches d'évaluation de l'impact de ces mêmes pratiques, pour l'instant cantonnée dans un registre exploratoire, mériterait d'être poursuivie.

Mots-clés : efficacité - typologies - processus d'enseignement/apprentissage - effet-classe - implication - interactions activité - acquisitions

\section{Abstract}

The present research aims at describing the efficiency of teaching practices invol-
- Nous renvoyons le lecteur à l'article de ces deux auteurs, publié dans cette même revue, consacré spécifiquement à l'appréhension d'une des modalités d'enseignement-apprentissage retenue par nos soins comme vecteur potentiellement pertinent des acquisitions des élèves, à savoir les interactions maître/élèves (ainsi qu'entre élèves).

- Voir l'introduction générale de ce numéro thématique. 
ved in dynamic "teaching-training" processes. Our main approach consisted in relating in-class-monitoring to the results of knowledge-acquisition by pupils so as to better explain through what means the pupils' knowledge and competence are acquired. At a theoretical level this implies combining the "process-product"paradigm along with the "ecological" paradigm [1].

Results obtained indicate that bringing together the analysis of teaching practices with the evaluation of their impact - which are still limited to an exploratory stage - are a method well-worth pursuing.

[1] See above the general introduction to this thematic issue

Key-words : Efficiency - typologies - teaching/training processes - in-class effect - implication - interactions - activity knowledge-acquisition 


\section{Cadre et enjeux de cette recherche}

Au plan de l'efficacité des pratiques d'enseignement que l'on regroupe classiquement en sociologie sous les labels "effets-classes et effets-maîtres" (Bressoux, 1994), on sait que "le poids" de la classe dans laquelle un élève est scolarisé ou du maître qui anime cette classe est loin d'être négligeable puisqu'il peut atteindre de l'ordre de $20 \%$ de

- "... quantification de la perte ou du gain pour un élève d'être scolarisé avec tel enseignant plưôt qu' avec tel autre..." (Bru, 2002 : 64) (on pourrait rajouter : dans telle classe plutôt que dans telle autre)

la variance des acquisitions des élèves. Pour autant, si cet impact est indubitable, les facteurs qui influent concrètement au sein de cet environnement pédagogique sur les acquisitions des élèves restent flous tant les variables liées à des approches quantitatives demeurent "grossières" (Bressoux, 2000) et/ou tant les méthodologies de recueil des données restent inadaptées à une perception fidèle et fine des phénomènes étudiés (la plupart du temps, on recourt uniquement à des questionnaires ). Ces constats ont étayé chacun de nos choix au fil d'une démarche qui se veut exploratoire.

Bien que d'autres recherches aient
montré combien les pratiques effectives
des enseignants pouvaient s'écarter de
leurs pratiques déclarées ! Voir par
exemple Delhaxhe A. (1997) sur la
question du temps consacré aux
différentes disciplines.

- Bien que d'autres recherches aient me combien les pratiques effectives leurs pratiques déclarées ! Voir par (1997) sur différentes disciplines.

La recherche que nous présentons ici a été initiée dans le cadre d'un projet plus vaste, consacré à l'expérimentation parallèle, dans cinq pays, d'un curriculum d'éveil aux langues au cycle 3 (élèves entre 8 et 11 ans) (Candelier (dir.), à paraître) et suscitée par lui. Il s'agissait, en effet, de tester empiriquement l'une des hypothèses du projet selon laquelle les effets attendus du programme en matière de développement d'aptitudes métalinguistiques et d'attitudes d'ouverture à la diversité linguistique et culturelle chez les élèves seraient d'autant plus importants si les enseignants mettaient en œuvre ces activités dans le cadre d'une pédagogie de type «pédagogie active », centrée sur l'élève (son activité, son implication...). Pour ce faire, nous avons par conséquent cherché, au-delà de la mesure des effets du programme sur les compétences visées chez les élèves, à mettre en œuvre un dispositif permettant de caractériser précisément les pratiques d'enseignement et d'apprentissage dans différentes classes expérimentales afin d'évaluer si certaines de ces pratiques se révélaient effective-

- Derrière cette notion floue de " pédagogie active » nous entendons des modèles d'enseignement / apprentissage au cœur desquels l'implication / participation / activité des élèves est fortement stimulée et entretenue et dont les processus à I'œuvre conduisent à une réelle coconstruction des savoirs et compétences. Au regard du curriculum d'éveil aux langues et de sa mise en actes, il serait d'ailleurs peut-être plus judicieux de parler de pédagogie "inter-active », voire « socioconstructiviste ». Cette perspective est notamment mise en valeur dans l'article de Aeby et de Pietro (ici même) lors de leur caractérisation des interactions maître-élèves. ment davantage efficaces que d'autres.

Il nous fallait donc conjointement nous inscrire dans le cadre d'une évaluation de type « processus-produits » et pouvoir recueillir des données suffisamment fines pour caractériser la plus ou moins grande proximité des pratiques enseignantes avec des modèles d'enseignement / apprentissage fondés sur l'implication / activité des élèves. A cette fin, nous avons choisi de privilégier l'observation des pratiques plutôt que de nous en référer aux seules déclarations des enseignants. Nous fondant sur divers travaux antérieurs qui mettent en évidence la diversité des pratiques effectives, même lorsque les enseignants font référence explicite à une 
méthode pédagogique semblable ou suivent des consignes explicites nous avons postulé que - malgré le fait que les supports didactiques proposés aux enseignants étaient tous structurés en référence à une théorie socioconstructiviste de l'apprentissage - les pratiques des enseignants allaient cependant pouvoir être différenciées.

Au-delà des résultats qui y sont présentés, notre contribution se veut essentiellement méthodologique, visant à proposer une réflexion sur les possibilités de mieux com-

- Bru (1992) montre par exemple à quel point les pratiques effectives des enseignants peuvent fluctuer autour de ce cadre de référence, notamment en adaptation aux situations d'enseignement et aux publics visés ; de même, Suchaut (1996) constate que, malgré des prescriptions contraignantes, le temps alloué au francais au CP peut varier du simple au double ! prendre l' « effet-classe » et de mettre en relation la construction effective de connaissances et la manière dont elles sont construites. Dans le cadre de cette approche nous ne cherchons pas à catégoriser ce que sont les pratiques enseignantes à l'œuvre mais, plutôt, ce que "font" les élèves, particulièrement en termes d'implication et d'activité. Les modalités de ce faire sont hypothétiquement supposées

- Le rôle des enseignants estici envisagé comme "vecteur" des dynamiques de classe et de la mise en activité des élèves. produire des effets tangibles au plan des acquisitions.

Par conséquent, le texte qui suit se propose de rendre compte des résultats obtenus par la mise en relation de deux dispositifs complémentaires :

- d'une part l'évaluation des progrès des élèves mesurée à travers la confrontation de tests initiaux et finaux portant sur les différentes compétences visées par le programme didactique - et dont l'analyse statistique de type « modélisation multivariée » permet de mettre en évidence, à côté des caractéristiques individuelles des élèves, l'impact des effets contextuels de scolarisation, encore appelés usuellement « effets-classe »;

- d'autre part l'observation et l'analyse approfondie, dans une vingtaine de classes de trois pays, de la manière dont les activités du programme expérimental ont été mises en œuvre dans les classes, afin de définir, par hypothèse et en référence au cadre théorique, des « fonctionnements » interactifs a priori plus efficaces que d'autres.

\section{La caractérisation des modalités d'enseignement-apprentissage}

Notre démarche d'analyse s'appuie sur un postulat qui consiste à considérer la classe comme une unité d'analyse pertinente au regard de l'efficacité du curriculum expérimental développé ; l'implication/activité des élèves y occupant une place que nous posons hypothétiquement comme centrale.

Durant cette phase nous avons choisi de ne pas prendre en compte les motivations pédagogiques des enseignants ni leurs choix pédagogiques sur un plan "formel", mais nous nous sommes centrés, en quelque sorte, sur la résultante de ces choix et motivations - fussent-ils inconscients - telle que l'on peut la saisir dans la mise en acte des situations d'enseignement-apprentissage, autrement dit dans le travail concret de l'enseignant d'un côté, dans les modes de participation et d'engagement des élèves de l'autre, puisque, pour nous, la classe constitue bien un espace de co-construction des savoirs. Pour ce faire, nous avons retenu trois angles 
d'observation donnant lieu à trois outils de recueil puis d'analyse que nous présenterons ci-après. Ces derniers mettent à nu autant "d'organisateurs" de la pratique d'enseignement (Clanet, 1997), "d'analyseurs" de la dynamique des classes.

Ils s'inspirent essentiellement des travaux antérieurs des équipes du CREN et du CREFI dont les savoirs-faire sont mobilisés notamment lors d'une étude exploratoire des pratiques d'enseignement en CE2 (Altet, Bressoux, Bru, Leconte-Lambert, 1994 et 1996a) (outils 1 et 2) ainsi que de l'analyse conversationnelle (issues des approches linguistiques, $c f$. : Roulet et al. 1985 ; Kerbrat-Orecchioni

- CREN : Centre de Recherche en Education de Nantes, Université de Nantes (responsable M. Altet) CREFI : Centre de Recherche en Education, Formation, Insertion, Université de Toulouse-Le Mirail (responsable M. Bru) 1990) au fondement de notre troisième outil créé spécifiquement pour les besoins de cette recherche ( $c f$. de Pietro et Aeby dans cette même revue).

\section{- Le dispositif d'observation des classes}

La lisibilité du protocole implique de donner quelques clefs de la constitution de l'échantillon des classes observées avant de présenter chacun des outils construits afin de caractériser les pratiques d'enseignement-apprentissage à l'œuvre dans nos classes expérimentales.

\section{L'échantillon de classes}

Trois pays sur les cinq impliqués dans la recherche-innovation support de cette analyse ont accepté de s'engager dans ce protocole, relativement lourd $₫$, comprenant différents types d'entretiens, un questionnaire-bilan, des cahiers de bord, un portfolio et des observations sous des angles croisés. L'échantillon ainsi constitué comprend une ving-

- Pour une présentation générale de la recherche dans laquelle notre expérimentation a pris place et du protocole utilisé dans ce cadre, voir Candelier [Dir.] (2003) et Genelot, S. \& Tupin F. (2003) taine de classes réparties en Espagne (Catalogne), en France métropolitaine, à l'île de La Réunion et en Suisse.

Les classes retenues pour mettre en œuvre ces observations l'ont été - autant que faire se peut - selon le principe de la diversité des "styles d'enseignement" telle qu'elle a pu être repérée lors des visites de classes ayant précédé l'observation systématisée. Ce critère s'inscrit dans la logique de mise à l'épreuve de notre hypothèse qui stipule que la variété des pratiques d'enseignement / apprentissage constituerait une source d'explication possible des différences de résultats observées entre les élèves.

Cet échantillon - pour lequel nous nous étions fixés, comme « point de repère », de toucher environ $15 \%$ de l'ensemble des classes expérimentales - tend également à prendre en compte la palette des différentes compositions sociologiques des classes réunies par cette expérience.

Pour cette partie de la recherche, 14 classes parmi les 20 observées ont finalement été retenues lors de nos traitements statistiques.

\section{Les dimensions des pratiques observées}

Rappelons que la confection de nos outils s'inscrit dans la mise à l'épreuve de l'une des hypothèses du projet 
EVLANG selon laquelle les effets du programme auprès de tous les élèves - y compris ceux qui sont scolairement les plus faibles - sont tributaires de leur implication et de leur activité, elles-mêmes en partie conditionnées par le type de démarche engagée par chaque enseignant.

Les observations visaient donc globalement à caractériser le déroulement des leçons. Mais il s'agissait aussi, par ailleurs, de réunir des conditions favorables à l'émergence du style pédagogique (Altet, 1993) de chaque enseignant concerné lorsqu'il interagit avec ses élèves. A cette fin, un support didactique moins « guidant », moins « ritualisé » que les autres supports du curriculum proposé - intitulé 1,2,3... 4000 langues - a été spécifiquement conçu, proposant une situation didactique ouverte apte à favoriser les initiatives des enseignants (et par là même une certaine « authenticité » qu'on suppose révélatrice d'un comportement pédagogique plus coutumier).

Ainsi, le dispositif d'observation a été mis en œuvre au cours de la même séance du curriculum dans toutes les classes concernées et ce, durant la même période de l'année scolaire sur chacun des sites. L'utilisation de trois outils complémentaires a permis d'analyser trois dimensions particulières du processus d'enseignement / apprentissage :

- la mesure de l'implication des élèves lors des phases de travail collectif de la séance (phase I dite de « mise en situation » et phase III dite de « synthèse-structuration ») ;

- les modes de communication entre élèves lors de la 2ème phase de la séance dont le déroulement prévoyait un travail en petits groupes (phase II, «Situation de recherche») ;

- enfin, l'analyse de la co-construction des contenus d'apprentissage dans les interactions entre élèves et enseignant lors de ces mêmes phases collectives.

Les deux premiers outils ("Communication interne" et "Implication") ont fait l'objet des premiers traitements sous forme d'un "tâtonnement expérimental" Nous nous contenterons ici d'en définir les principes et les modalités principales (voir 1.2.1 et 1.2.2). Le troisième outil a fait l'objet d'un traitement ultérieur en raison de sa complexité. Il est présenté de façon

- Ils ont été présentés in extenso lors d'une communication à laquelle nous renvoyons le lecteur intéressé par cet aspect de notre travail (Genelot et Tupin, Lille 2001). détaillée dans le cadre de ce numéro thématique (cf. l'article cosigné par JF. de Pietro et $\mathrm{S}$. Aeby). Il sera donc mentionné ci-après de façon relativement synthétique (voir 1.2.3)

- Voir également, de Pietro et al, (2003)

\section{- 1.2 Les différentes typologies des modalités d'enseignement-apprentissage}

La présentation de ces trois outils emprunte un schéma récurrent qui adopte la structure suivante : présentation de l'outil et de ses visées, synthèse des différents indicateurs retenus, typologie des classes qui résulte de la mise en œuvre de l'outil. Ces trois outils de recueil/analyse visent, chacun dans une logique particulière, à réifier la dynamique des échanges en classe, que ceux-ci soient verbaux ou non. 


\section{I.2.1. L'implication des élèves}

Si l'implication des élèves dans l'activité constitue, selon la terminologie de Altet et al. (1996b), une "micro-variable", elle a, pour nous, valeur de "macro-analyseur" en ce sens qu'elle permet de reconstituer la dynamique des classes dans leur temporalité, à chacune des phases, mais aussi de façon globale.

L'outil de recueil utilisé s'inspire largement des travaux de Altet, Bressoux, Bru et Leconte-Lambert (1994 et 1996a), il a ensuite été adapté à nos objectifs et contraintes propres d'observation. Il consiste à observer trois élèves-types (un "fort", un "moyen", un "faible") sélectionnés, pour chaque classe, sur la base des indications du maître. Cette observation de l'implication de trois élèves-types s'inscrit durant toute la durée de la séquence didactique observée. Elle s'opère dans une rotation continue des observations baptisée "carrousel de l'implication".

- (On regarde l'élève de « niveau scolaire faible », on évalue s'il est impliqué ou non, on relève ; on fait de même pour l'élève « de niveau scolaire moyen », puis pour celui de « niveau scolaire fort 》 et ainsi de suite.)

Pour résumer les phénomènes étudiés, on peut dire que ce qui est le plus difficile pour un enseignant consiste à impliquer les élèves de niveau scolaire faible, à les impliquer tout au long du déroulement de la séance, et à les impliquer à un niveau comparable à celui des élèves de « bon » niveau scolaire (soit 3 "niveaux" de difficulté). On peut donc supposer que les dynamiques de classe qui conduisent à résoudre au mieux cette équation sont, hypothétiquement, porteuses d'efficacité au plan des acquisitions des élèves.

L'exploitation/analyse de ces matériaux s'inscrit dans la prise en compte des trois niveaux de difficulté évoqués précédemment. Elle conjugue ainsi cinq critères principaux :

- l'implication de chaque élève au cours de chacune des trois phases du canevas didactique ;

- le coefficient moyen d'implication de chaque "élève-type" tout au long de la séance ;

- le degré d'équilibre ou de déséquilibre du niveau d'implication entre élèves de niveaux scolaires contrastés (et ce à chaque phase) ;

- la hiérarchie de la mobilisation en fonction des niveaux scolaires ;

- et, enfin, l'évolution de cette mobilisation au fil du déroulement de la séance observée.

A terme, ces critères donnent lieu à un coefficient qui synthétise, pour chaque classe, le "degré de mobilisation des élèves autour de l'activité". C'est sur la base de ce coefficient que la typologie des classes est établie.

Les classes observées s'y répartissent ainsi :

- Les catégories sont utilisées lors de nos présentations des traitements statistiques (voir ci-après le tableau 3).

Typologie des "implications".

\begin{tabular}{|lcccc|}
\hline $\begin{array}{l}\text { Classes } \mathrm{n}^{\circ} \\
\text { Types } \\
\text { hiérarchisés } \\
\text { attendus }\end{array}$ & $\begin{array}{c}\text { Profils d'implications } \\
\text { "a priori" les + favorables } \\
\text { aux apprentissages }\end{array}$ & $6 ; 7 ; 15$ & $10 ; 11 ; 16 ; 17$ & $2 ; 3 ; 8$ \\
\hline Catégories" $^{4}$ & 1 & 2 & $\begin{array}{c}\text { Profils d'implications } \\
\text { "a priori" les - favorables } \\
\text { aux apprentissages }\end{array}$ \\
\hline
\end{tabular}




\subsubsection{La communication des élèves dans les groupes}

Il nous a semblé que ce moment spécifique de travail en petits groupes (de 3 à 5 élèves) autour d'une "situation de recherche" (phase II du canevas didactique) pouvait constituer un moment privilégié de co-construction des connaissances ; cette co-construction "entre élèves" étant accompagnée par les interventions de l'enseignant (type de consignes données et type de guidage). Durant cette étape d'investigation, nous cherchons par conséquent à percevoir quels sont les éléments constitutifs de la dynamique de la communication au sein de chaque groupe d'élèves.

Plus précisément, on cherche à savoir comment les élèves :

- s'approprient la démarche impulsée/relancée/guidée par le maître et/ou induite par le support,

- s'impliquent dans la tâche, participent...

- gèrent la formulation d'hypothèses, la résolution de problèmes...

- gèrent leur marge d'autonomie, etc.

L'hypothèse sous-jacente étant que : plus cette communication est autonome, fluide, équilibrée et partagée entre les élèves, quels que soient leurs niveaux scolaires, et plus les résultats / acquisitions liés à la démarche d'éveil aux langues devraient être positifs.

Afin de limiter les biais dus à l'interprétation des observateurs, deux sous-groupes ont, dans chaque classe, été observés en parallèle.

Les unités d'analyses retenues pour mener l'observation se répartissent en 7 champs (regroupant au total 25 items) :

- disposition spatiale ;

- rôle de l'enseignant (type de consigne et type de guidage) ;

- initiative de la répartition des rôles des élèves ;

- dynamique du groupe ;

- leadership / relation de pouvoir ;

- prises de décisions ;

- centration sur l'élève en difficulté.

Pour l'exploitation/analyse de nos corpus, nous nous sommes astreints à un retour sur nos données à partir de vidéos tournées durant les séances. Les critères retenus ont alors été pondérés. De plus, en référence à notre hypothèse, deux indices agrégés ont été construits afin de focaliser l'analyse sur la possibilité donnée à tous les élèves d'être « actifs ». Le premier indice concerne l'activité / fonctionnement des élèves dans le groupe, de façon générale. Le second se centre sur le rôle de l'élève repéré comme « scolairement faible » dans cette dynamique de groupe.

L'ensemble de ces arbitrages donne lieu à une typologie des modes de communication interne dominants dans les différentes classes observées regroupées par profils. Les classes de notre échantillon retenues dans nos analyses s'y répartissent de la façon suivante :

- Les catégories sont utilisées lors de nos présentations des traitements statistiques (voir ci-après le tableau 3). 
Typologie des " modes de communication interne".

\begin{tabular}{|lccccc|}
\hline Classes $^{\circ}$ & $7 ; 9$ & $8 ; 11 ; 12 ; 17$ & $4 ; 5$ & $6 ; 10 ; 16$ & $2 ; 3 ; 15$ \\
\hline $\begin{array}{l}\text { Types } \\
\text { hiérarchisés } \\
\text { attendus }\end{array}$ & $\begin{array}{l}\text { Profils de communication } \\
\text { "a priori" les + favorables } \\
\text { aux apprentissages }\end{array}$ & & & & $\begin{array}{l}\text { Profils de communication } \\
\text { "a priori" les - favorables } \\
\text { aux apprentissages }\end{array}$ \\
\hline Catégories $^{4}$ & 1 & 2 & 3 & 4 & 5 \\
\hline
\end{tabular}

\subsubsection{Les interactions enseignant/élèves et entre élèves}

Dans le cours de l'élaboration d'outils qui nous permettraient de cerner différentes dimensions caractérisant les modalités d'enseignement / apprentissage dans les classes, il nous est rapidement apparu qu'il fallait tenir

- Ainsi que nous l'avons signalé, l'outil "interaction" fait l'objet, dans ce même numéro, d'une large présentation dans le texte de S. Aeby. et JF. de Pietro compte de l'apport des diverses activités discursives de l'enseignant et des élèves à la construction des contenus d'apprentissage, autrement dit de la manière dont ces contenus sont (co-)construits par les partenaires dans le cours des échanges en classe : qui apporte les contenus d'apprentissage ? quelle proportion des élèves participe à cette construction ? que fait l'enseignant pour aider les élèves dans leur travail de construction?...

Ce faisant, nous avons cherché à comprendre la construction interactive des contenus tout en prenant en compte quelques éléments de cette construction qui constitueraient des spécificités de l'approche de type éveil aux langues dans laquelle nous avons travaillét.

- Afin de généraliser notre propos, cet aspect de l'analyse ne sera pas envisagé dans cet article. Cf. de Pietro et al. (2003) et Candelier [Dir.] (2003), chapitres 7 et 8 .

Cela nous a conduit à définir, après de nombreux tâtonnements, 9 indicateurs qui nous semblent des éléments importants dans la caractérisation du type de pédagogie mise en œuvre :

1. DIV : proportion d'élèves différents qui participent discursivement à la construction des contenus d'apprentissage ;

2. QUANT : temps de parole des élèves par rapport à celui de l'enseignant ;

3. ACT : engagement des élèves, tel qu'il est exprimé par une volonté manifeste d'intervenir (demande de prise de parole, prise de parole sans demande préalable...);

4. SOCIO : construction interactive des contenus et étayage de l'enseignant ;

5. CONT1 : apport respectif des élèves et de l'enseignant à la formulation des contenus d'apprentissage ;

6. CONT2 : qualité et quantité des contenus traités durant la leçon ;

7. GUID : guidance de l'enseignant, telle qu'elle est exprimée par la nature plus ou moins contraignante de ses initiatives ;

8. OUV : place accordée aux apports des élèves et à la diversité des langues (dimension fortement liée aux principes des démarches d'éveil aux langues);

9. SCOL : utilisation de ressources (dictionnaires, cartes géographiques,...), établissement de liens avec les autres disciplines scolaires, qui marquent l'intégration des démarches d'éveil aux langues dans les pratiques scolaires ordinaires.

Pour chacun de ces indicateurs, des catégories ont été établies, empiriquement, sur la base des options didactiques prises dans le cadre du programme Evlang et de 
nos hypothèses de recherche. Par exemple, l'indicateur CONT1 a été distribué en 4 classes définies en fonction du rapport entre le nombre d'apports significatifs des élèves et le nombre d'apports significatifs de l'enseignant :

- rapport $<2$ : indice - -

- rapport entre 2 et 4 : indice -+

- rapport entre 4 et 9 : indice +-

- rapport $>9$ : indice ++ .

De manière générale, en liaison avec nos hypothèses, l'indice «++ » est considéré comme potentiellement favorable aux apprentissages, l'indice « - - comme potentiellement défavorable.

Plusieurs typologies peuvent être établies à partir de données aussi nombreuses et diversifiées. Il est par exemple possible d'établir un classement pour chaque indicateur et mesurer ceux qui s'avèrent les plus efficaces pour expliquer la progression des élèves dans les compétences évaluées . La typologie que nous avons exploitée ici vise à prendre en compte l'ensemble des indicateurs en un seul classement. Elle a été construite à partir d'une exploitation « arithmétique » des données : nous avons en effet

- Une telle analyse est présentée dans les actes du colloque «Construction des connaissances et langage dans les disciplines d'enseignement ", Bordeaux 3-5 avil 2003, voir en bibliographie De Pietro, J.F ; Aeby, S. ; Genelot, S. ; Tupin, F. (2003) choisi de ne pondérer aucun des indices des neuf indicateurs utilisés pour l'analyse des interactions en attribuant une valeur de 1 au signe « + » et une valeur de -1 au signe « - », et en orientant les classes qui se trouvent à cheval sur deux groupes selon qu'elles obtiennent davantage de « +/- » ou de « -/+ ».

Cette option revient à conserver toutes les dimensions des interactions observées et à leur attribuer le même « poids potentiel » au plan des acquisitions des élèves (et donc de l'efficacité de l'enseignement dispensé). Ou plutôt, elle revient à reconnaître que nous sommes dans l'impossibilité, à l'heure actuelle, de démêler de façon pertinente cet "écheveau" ainsi que de contrôler les interdépendances entre les différents paramètres observés.

Plus positivement, cette option exprime, selon nous, un impératif d'équilibre dans la mise en œuvre des différentes composantes de la relation pédagogique afin d'approcher un schéma d'enseignement / apprentissage optimal.

En appliquant cette logique, nous parvenons à la typologie suivante issue de la combinaison des 9 indicateurs présentés ci-avant :

- Les catégories sont utilisées lors de nos présentations des traitements statistiques (voir ci-après le tableau 3).

Typologie des "interactions".

\begin{tabular}{|c|c|c|c|c|}
\hline Classes $n^{\circ}$ & $5 ; 6 ; 12 ; 15$ & $7 ; 9 ; 17$ & $4 ; 16$ & $2 ; 3 ; 8$ \\
\hline $\begin{array}{l}\text { Types } \\
\text { hiérarchisés } \\
\text { attendus }\end{array}$ & $\begin{array}{l}\text { Profils d'interaction } \\
\text { "a priori" les + favorables } \\
\text { aux apprentissages }\end{array}$ & & & $\begin{array}{l}\text { Profils d'interaction } \\
\text { "a priori" les - favorables } \\
\text { aux apprentissages }\end{array}$ \\
\hline Catégories" & Intl & Int2 & Int3 & $\operatorname{lnt} 4$ \\
\hline
\end{tabular}

Deux classes n'ont par ailleurs pas pu être intégrée dans cette typologie, les classes 10 et 11 , en raison du fait du fait qu'il manque certaines données à leur 
sujet pour quelques indicateurs. Elles formeront, lors des traitements, une catégorie à part (Intinc).

Sur la base de ce regroupement des classes en 4 (5 si l'on prend en compte la catégorie Inconnu) différents groupes, nous nous attendons donc, en particulier, à ce que les élèves des classes figurant dans le groupe 1 - qui bénéficient de la dynamique d'enseignement-apprentissage "la plus interactive" et donc a priori la plus favorable aux acquisitions - progressent davantage que ceux du groupe 4 .

\section{L'évaluation de l'efficacité des pratiques enseignantes}

Outre le dispositif d'observation des classes de l'échantillon qui vient d'être exposé, le dispositif de recherche comprenait également l'évaluation, à l'aide de tests, de la progression des élèves avant et après la mise en œuvre du programme EVLANG dans ces classes (soit 18 mois plus tard, ce qui correspond à une période d'enseignement / apprentissage effective de 13 mois) à l'aide de tests. Dans une première partie, nous présenterons les variables qui ont été construites à partir de ces épreuves

- Pour une présentation détaillée du programme Evlang et des principes de "l'eveil aux langues" voir : Candelier, 1998 et Candelier [Dir.], 2003. Voir également l'article de Aeby et de Pietro ici même. ainsi que le mode d'analyse utilisé pour traiter l'ensemble des données. Dans un second temps nous rendrons compte des résultats de la mise en relation des modalités d'enseignement-apprentissage appréhendées sous la forme des typologies de classes (présentées dans la partie I) avec la progression des élèves.

\section{- II.1 Le cadre méthodologique d'évaluation}

\section{II.1.1 Les aptitudes évaluées}

Les épreuves destinées à mesurer la progression des élèves dans les différents domaines concernés par le programme expérimental EVLANG ont été construites à partir du corpus d'hypothèses sur les effets attendus auprès des élèves.

Il a donc fallu chercher, dans un premier temps, comment pouvait être opérationnalisée l'évaluation des aptitudes et attitudes que le programme EVLANG cherche à développer chez les élèves et élaborer, pour ce faire, des épreuves originales dans le sens où elles concernent des acquisitions relativement peu présentes dans les programmes officiels de l'école primaire des cinq pays concernés

- Pour une présentation du contenu de ces épreuves, cf. Candelier [Dir.], 2003, chapitre 4.

L'exploitation des données issues de ces épreuves a conduit à la construction de douze variables dépendantes visant à exprimer les performances des élèves sous une forme synthétique. Ce sont ces variables dont il s'agit d'expliquer la variété. Dans le cadre de cet article nous ne présenterons que les deux variables qui concernent les aptitudes métalinguistiques des élèves. En effet, le caractère exploratoire de ce travail nous a amenés à nous en tenir, dans un premier temps, à un nombre réduit de compétences analysées. Les deux variables retenues ont été choisies sur deux critères principaux : d'une part le fait que le programme EVLANG se soit révélé particulièrement efficace pour faire progresser les élèves 
dans ces domaines de compétences, d'autre part parce que nous pensions qu'elles pouvaient se révéler plus sensibles que les autres (notamment que celles relevant de l'évolution des attitudes des élèves) aux variations des pratiques pédagogiques mises en œuvre dans les classes.

Les épreuves portant sur les aptitudes métalinguistiques des élèves dans des langues non familières avaient pour objet d'explorer les dimensions suivantes : l'habileté à repérer et distinguer des sons dans une langue non familière (exercices principalement oraux); l'habileté à combiner des morphèmes dans une langue non familière (à l'écrit); l'habileté à déduire des règles syntaxiques par l'observation d'énoncés en langue non familière (à l'écrit)
- Dans le texte, nous avons parfois recours à une terminologie de type « oral » versus "écrit 》 pour désigner ces deux variables. Les aptitudes en jeu, cependant, ne concernent pas exclusivement l'un ou l'autre mode. L'emploi de ces expressions n'a donc que des visées simplificaticices pour l'économie générale du texte.

A partir de ces activités, nous avons construit deux variables de scores différentes :

DECOMP : décomposition et recomposition (à l'écrit) d'énoncés dans différentes langues non familières ;

MEMDISC : mémorisation et discrimination auditive dans différentes langues non familières.

Le tableau ci-après présente le contenu des activités retenues pour ces deux variables :

\begin{tabular}{|c|c|}
\hline \multicolumn{2}{|r|}{ Variable DECOMP : somme des scores obtenus aux activités suivantes: } \\
\hline Activité 2 & $\begin{array}{l}\text { La tâche consiste à observer une liste de nombres en chinois (pour le test initial) et en finnois (pour le } \\
\text { test final) pour en déduire les règles de formation de la numération dans ces langues. L'èlève doit donner } \\
\text { l'équivalent en chiffres de trois nombres écrits en lettres et l'équivalent en lettres de deux nombres } \\
\text { chiffrés. }\end{array}$ \\
\hline Activité 5 & $\begin{array}{l}\text { En observant comment sont construits } 3 \text { énoncés écrits dans une langue non familière dont la traduction } \\
\text { est donnée en francais, l'élève doit déduire la forme d'un quatrième énoncé (traduction d'un énoncé en } \\
\text { langue de l'école dans cette langue non familière). Le repérage est ici d'ordre morphologique } \\
\text { (combinaison de morphèmes). }\end{array}$ \\
\hline Activité 6 & $\begin{array}{l}\text { La tâche contenue dans cet item est du même ordre que celle de l'activité précédente mais le r } \\
\text { fait cette fois aux deux niveaux morphologique et syntaxique (ordre des mots à respecter). }\end{array}$ \\
\hline \multicolumn{2}{|r|}{ Variable MEMDISC : somme des scores obtenus aux activités suivantes : } \\
\hline Activité 1 & La tôche consiste à écouter l'énoncé d'un mot-cible et à le repérer dans une série de trois mots. \\
\hline Activité 3 & $\begin{array}{l}\text { La tâche consiste à identifier un locuteur et une locutrice parlant la même langue non familière. L'élève } \\
\text { entend tout d'abord un enregistrement de quelques secondes d'une personne, puis } 3 \text { enregistrements de } \\
\text { trois personnes du sexe opposé. Une des langues entendues est la même que celle du premier } \\
\text { enregistrement, l'élève doit l'identifier. }\end{array}$ \\
\hline Activité 4 & $\begin{array}{l}\text { La tâche consiste à apparier deux items présentant des caractéristiques semblables, soit au niveau } \\
\text { phonétique, soit au niveau prosodique (repérage des tons dans des mots monosyllabiques en chinois, par } \\
\text { exemple). L'élève entend un premier item, puis deux autres. II doit apparier le premier item à celui qui lui } \\
\text { ressemble le plus dans le groupe des deux items. }\end{array}$ \\
\hline
\end{tabular}

Tableau 1. Construction des deux variables DECOMP et MEMDISC

\section{II.1.2 Le mode de traitement des données}

Le traitement des données a été réalisé au moyen d'analyses de régression multivariée. Le principe de ces analyses statistiques repose sur l'explication de la 
variété des acquisitions des élèves au test final sous la forme d'une relation mathématique entre le niveau de performance des élèves et l'ensemble des facteurs que l'on peut et que l'on choisit « d'objectiver» (caractéristiques individuelles des élèves, caractéristiques pédagogiques des classes...).

Ici les deux variables DECOMP et MEMDISC constituent les variables dépendantes dont on cherche à expliquer la variété. Le fait d'appartenir à une classe de type 1, 2 ou 3 (modalités des typologies d'enseignement-apprentissage retenues) est alors considéré comme une caractéristique particulière de chaque élève de l'échantillon. L'utilisation de la modélisation multivariée va permettre de mesurer l'impact spécifique de cette « variable-cible » sur le niveau d'acquisition final des élèves, indépendamment de l'influence des autres facteurs (c'est à dire « toutes choses égales par ailleurs »). Pour ces derniers, nous disposons, par le biais d'un questionnaire, d'un certain nombre de renseignements concernant les élèves (âge, sexe, profession de leur parents) (CE) ainsi que leur niveau initial (AI), constituant les variables explicatives dites « de contrôle ».

On peut résumer ce type d'analyse sous la forme de l'équation générale suivante :

$$
\mathrm{AF}=\mathrm{f}(\mathrm{AI}, \mathrm{CE}, \mathrm{TP})
$$

$\mathrm{AF}$ : niveau d'acquisition final

$\mathrm{AI}$ : niveau d'acquisition initial

$\mathrm{CE}$ : caractéristiques des élèves

$\mathrm{TP}$ : type de pratiques pédagogiques observées.

\section{- II.2 Evaluer l'efficacité des modalités d'enseignement- apprentissage}

La mise en relation des modalités d'enseignement-apprentissage avec la progression des élèves dans les deux domaines de compétences concernés a été conduite en deux phases successives. Dans un premier temps nous avons mesuré l'ampleur des "effets classes" dans l'échantillon et nous avons tenté une première analyse de type exploratoire en rapprochant la hiérarchie d'efficacité des classes obtenue de la répartition des classes dans les différentes typologies d'enseignement-apprentissage. L'idée générale était d'essayer de repérer des tendances dans la relation entre efficacité et "types" de pratiques, lesquelles devaient être confirmées ou infirmées par la seconde phase d'analyse qui a consisté à intégrer les typologies des pratiques observées dans les modèles multivariés comme variables explicatives de la progression des élèves.

\section{II.2.1 Hiérarchie d'efficacité des classes et typologies de pratiques d'enseignement-apprentissage : approche exploratoire}

Plusieurs modèles successifs ont donc été estimés pour mesurer l'impact des différents facteurs pris en compte dans nos analyses sur les compétences métalinguistiques des élèves. Le premier modèle n'intègre que le seul niveau initial des élèves dans la compétence évaluée comme variable explicative du niveau final atteint. Le second modèle intègre, en plus du niveau initial, les caractéristiques individuelles des élèves. Enfin, le troisième modèle, dit « des effets classes », permet d'intégrer dans l'analyse la classe dans laquelle chacun des élèves est scolarisé. 


\section{A) L'ampleur des effets-classe dans l'échantillon}

Le principal intérêt de ces trois modèles est dans un premier temps de permettre de comparer le poids respectif des différents groupes de variables dans l'explication de la variété des acquisitions des élèves dans les deux aptitudes évaluées. C'est ce que met en relief la comparaison des coefficients R2 des différents modèles $\$$, présentés dans le tableau ci-dessous.

- Le coefficient R2 indique le pourcentage de variance de la variable dépendante du modèle (ici le score final des élèves dans les deux aptitudes évaluées) expliquée par l'ensemble des variables explicatives prises en compte dans le modèle.

\begin{tabular}{|l|cc|}
\hline \multirow{2}{*}{$\begin{array}{l}\text { \% de variance expliquée } \\
\text { par chaque modèle }\end{array}$} & \multicolumn{2}{|c|}{ Aptitudes Métalinguistiques } \\
\cline { 2 - 3 } & $\begin{array}{c}\text { Tâches de décomposition } \\
\text { et recomposition à l'écrit d'énoncés } \\
\text { en langue étrangère } \\
\text { (DECOMP) }\end{array}$ & $\begin{array}{c}\text { Tâches de mémorisation } \\
\text { et de discrimination } \\
\text { auditives en langues étrangères } \\
\text { (MEMDISC) }\end{array}$ \\
\hline $\begin{array}{l}\text { Modèle 1 } \\
\text { Niveau initial (NI) }\end{array}$ & $15.8 \%$ & $2.5 \%$ \\
\hline $\begin{array}{l}\text { Modèle 2 } \\
\mathrm{Nl}+\text { Caractéristiques Elèves (CE) }\end{array}$ & $26.5 \%$ & $14.6 \%$ \\
\hline $\begin{array}{l}\text { Modèle 3 } \\
\mathrm{NI}+\text { CE + Classes } \\
\text { (gain par rapport au modèle 2) }\end{array}$ & $34.5 \%$ & $32.9 \%$ \\
\hline
\end{tabular}

Tableau 2. Pouvoir explicatif des modèles expliquant la variété des progressions des élèves dans les deux domaines d'acquisition concernés par les différents groupes de variables

On constate que les deux aptitudes évaluées chez les élèves ne sont pas également « sensibles » aux différents facteurs explicatifs pris en compte ici. Si ces compétences apparaissent à peu près également socialement déterminées (entre 10 et $12 \%$ de la variété des scores expliquée par les caractéristiques individuelles des élèves), on observe par ailleurs que les aptitudes relevant de la mémorisation et de la discrimination auditive sont davantage sensibles au contexte des classes alors que celles relevant de la décomposition/recomposition d'énoncés apparaissent surtout déterminées par le niveau initial des élèves dans ce domaine. On peut faire l'hypothèse que les premières sont peu ciblées (donc a priori peu travaillées en classe) par les programmes de l'école primaire et sont donc plus dépendantes de ce qui a réellement été travaillé au cours de la période d'expérimentation du programme EVLANG. A l'inverse, les épreuves proposées pour mesurer les aptitudes réunies sous DECOMP sont plus proches des objectifs visés par les programmes scolaires dans le domaine de la langue de l'école. Il n'est donc pas étonnant de trouver que le niveau initial des élèves dans ce domaine pèse de façon plus importante que pour le premier type d'acquisition.

En tout état de cause, on constate surtout que le poids du contexte global de la classe sur les acquisitions des élèves est loin d'être marginal (8\% pour DECOMP et $18 \%$ pour MEMDISC). Reste à savoir si les dimensions de la relation pédagogique que nous allons prendre en compte dans nos analyses à partir des données issues des observations des classes contribuent à expliquer - et dans quelle mesure - cet impact du contexte de scolarisation sur la progression des élèves. 


\section{B) Efficacité des classes et modalités d'enseignement-apprentissage}

Le tableau ci-après met en relation la hiérarchie de l'efficacité des classes obtenue à la suite de l'estimation des modèles de type 3 , pour chacune des deux aptitudes évaluées, avec la répartition de ces classes dans les différentes typologies d'enseignement-apprentisage retenues.

Notons premièrement que pour les compétences métalinguistiques relevant de la décomposition/recomposition d'énoncés (DECOMP), peu de classes (5) présentent une efficacité significative alors qu'elles sont plus nombreuses dans ce cas (10) pour les compétences relevant de la discrimination et de la mémorisation auditives (MEMDISC). Ce constat ne nous étonne guère compte tenu des observations faites précédemment concernant le pourcentage de variance des acquisitions expliqué par les différences inter-classes, beaucoup plus important pour MEMDISC que pour DECOMP.

\begin{tabular}{|c|c|c|c|c|c|c|c|}
\hline & \multicolumn{4}{|c|}{ Hiérarchie d'efficacité des classes } & \multicolumn{3}{|c|}{$\begin{array}{c}\text { Typologie des pratiques } \\
\text { d'enseignement-apprentissage } 0\end{array}$} \\
\hline & DEC & & MEMD & & $\begin{array}{l}\text { Communication } \\
\text { interne dans les } \\
\text { groupes d'élèves }\end{array}$ & $\begin{array}{l}\text { Implication } \\
\text { des élèves }\end{array}$ & $\begin{array}{c}\text { Profil } \\
\text { interactionnel } \\
\text { de la classe }\end{array}$ \\
\hline & Coef & Rang & Coef & Rang & Catégorie & Catégorie & Catégorie \\
\hline Classe 2 & -5.3 & & +3.6 & 4 & 5 & 4 & 4 \\
\hline Classe 3 & -5.3 & & -11.3 & & 5 & 4 & 4 \\
\hline Classe 4 & -5.3 & & -2.0 & 10 & 3 & 1 & 3 \\
\hline Classe 5 & +11.1 & 1 & +2.0 & 7 & 3 & 1 & 1 \\
\hline Classe 6 & -5.3 & & +7.9 & 3 & 4 & 2 & 1 \\
\hline Classe 7 & -5.3 & & -11.3 & & 1 & 2 & 2 \\
\hline Classe 8 & -5.3 & & +2.0 & 7 & 2 & 4 & 4 \\
\hline Classe 9 & -5.3 & & +8.3 & 2 & 1 & 1 & 2 \\
\hline Classe 10 & -5.3 & & -11.3 & & 4 & 3 & 2 \\
\hline Classe 11 & +10.1 & 2 & +3.6 & 4 & 2 & 3 & \\
\hline Classe12 & +6.4 & 5 & +1.2 & 9 & 2 & 1 & 1 \\
\hline Classe 15 & +7.1 & 4 & -11.3 & & 55 & 2 & 1 \\
\hline Classe 16 & -5.3 & & +16.7 & 1 & 4 & 3 & 3 \\
\hline Classe 17 & +9.5 & 3 & +2.2 & 6 & 2 & 3 & 2 \\
\hline
\end{tabular}

Tableau 3. Récapitulatif des hiérarchies d'efficacité des classes obtenues pour chaque aptitude et des typologies des classes obtenues suite à l'observation des pratiques enseignantes.
- Le principe utilisé consiste à construire autant de variables muettes que de classes de l'échantillon: une d'entre elle est mise en référence (par convention, il s'agit de la classe dont la moyenne est la plus faible), les autres sont introduites dans le modèle de base. On obtient ainsi une hiérarchie de l'efficacité des classes pour chaque aptitude évaluée qui se traduit par un coefficient (écart à la moyenne de l'efficacité de l'ensemble des classes: la somme de ces écarts étant égale à 0) qui permet de définir le rang de chaque classe dans la hiérarchie. Les classes dont le coefficient est positif sont les classes qui sont plus efficaces que la moyenne, celles dont le coefficient est négaifif sont moins efficaces que la moyenne. Pour les classes dont l'efficacité est non significative par rapport à lo classe de référence, leur coefficient est égal à celui de cette dernière ; c'est ce qui explique que dans le tableau ci-après, on trouve plusieurs classes avec des coefficients identiques (ce sont les classes qui, bien que ne se distinguant pas entre elles du point de vue de leur efficacité dans le domaine concerné sont néanmoins les classes les moins efficaces de l'échantillon). 
Un second constat s'impose : une même classe peut présenter une efficacité différenciée selon les deux types d'aptitudes métalinguistiques considérés ici. Ainsi, la classe 16 est la plus performante dans les tâches de mémorisation/discrimination auditives alors qu'elle est une des classes les moins performantes pour les tâches de décomposition/recomposition. Le phénomène inverse est observé pour la classe 15 , performante dans les compétences « à l'écrit », elle est parmi les moins performantes pour les compétences « orales ». Seules quelques classes peuvent être repérées comme étant significativement efficaces pour les deux compétences à la fois (classes 5,11, 12 et 17). Ce constat constitue en lui même un résultat intéressant : le travail des capacités « DECOMP » et des capacités « MEMDISC » demanderait-il des modalités différentes de fonctionnement dans la classe ?

A titre exploratoire, essayons maintenant de tester le caractère prédictif d'efficacité des trois typologies de pratiques en présence en rapprochant les profils d'efficacité des classes de leur position dans les typologies des pratiques d'enseignement-apprentissage. On peut tout d'abord regarder du côté des classes qui sont "bien placées" dans les trois typologies (condition a priori la plus favorable aux acquisitions des élèves).

L'exemple de la classe 12 aurait tendance à conforter notre hypothèse, placée en 1ère catégorie à la fois en matière d'implication des élèves et du point de vue des interactions et en seconde catégorie pour la communication interne dans les groupes, elle se trouve être particulièrement efficace en ce qui concerne les tâches de décomposition/recomposition d'énoncés (DECOMP) ainsi (quoiqu'un peu moins fortement) qu'en mémorisation et discrimination auditives (MEMDISC). Ceci dit la relation paraît moins simple concernant la classe 9 puisque malgré des pratiques enseignantes a priori favorables (catégorie 1 pour "communication interne" et pour "implication" et catégorie 2 pour "interactions") elle apparaît certes très efficace « à l'oral » mais pas du tout à « l'écrit ». Ces observations semblent conforter l'intuition exprimée plus haut selon laquelle certaines acquisitions seraient plus sensibles à certaines dimensions pédagogiques qu'à d'autres.

A l'extrême inverse - celui des classes «mal placées » dans les trois typologies - on peut, là encore, trouver une classe qui conforte nos attentes. C'est le cas de la classe 3 placée dans la dernière catégorie pour les trois typologies et qui s'avère, dans le même temps, particulièrement peu efficace dans les deux domaines d'acquisitions ciblés. Cependant là encore on trouve des situations plus contrastées. Les classes 2 et 16 plutôt "mal placées", elles aussi, dans les trois typologies de pratiques apparaissent cependant très efficaces pour faire progresser les élèves en mémorisation et discrimination auditives.

Au terme de ces observations on constate donc que la relation entre les deux types de hiérarchies - celle de l'efficacité et celles des profils de pratiques - est loin de pouvoir être établie par ce type d'approche, même si quelques cas extrêmes pourraient sembler la confirmer. L'intégration des typologies des pratiques observées, dans les modèles multivariés comme variables explicatives de la progression des élèves, va nous permettre d'examiner de façon plus fiable cette relation. 


\section{II.2.2 Les typologies de pratiques d'enseignement-apprentissage comme variables explicatives de la progression des élèves: approche intégrée}

Le tableau ci-dessous présente les résultats des modèles dans lesquels les trois typologies des pratiques d'enseignement-apprentissage ont été intégrées, en plus du niveau initial des élèves et de leurs caractéristiques individuelles, pour expliquer la variété des acquisitions des élèves dans les deux domaines considérés.

\begin{tabular}{|c|c|c|c|c|c|c|}
\hline & \multicolumn{6}{|c|}{ Aptitudes Métalinguistiques } \\
\hline & \multicolumn{3}{|c|}{$\begin{array}{c}\text { Tâches de décomposition et recomposition } \\
\text { à l'écrit d'énoncés en langue étrangère } \\
\text { DECOMP }\end{array}$} & \multicolumn{3}{|c|}{$\begin{array}{c}\text { Tâches de mémorisation et de discrimination } \\
\text { auditives en langues étrangères } \\
\text { MEMDISC }\end{array}$} \\
\hline & $\begin{array}{c}\text { Modalité } \\
\text { en référence } 1\end{array}$ & $\begin{array}{c}\text { Modalités } \\
\text { actives }\end{array}$ & $\begin{array}{l}\text { Gain en } \% \\
\text { de variance } \\
\text { expliquée?2 }\end{array}$ & $\begin{array}{c}\text { Modalité } \\
\text { en référence }\end{array}$ & $\begin{array}{c}\text { Modalités } \\
\text { actives }\end{array}$ & $\begin{array}{l}\text { Gain en \% } \\
\text { de variance } \\
\text { expliquée }\end{array}$ \\
\hline $\begin{array}{l}\text { Modèle } 1 \\
\text { Implication } \\
\text { des élèves }\end{array}$ & il & $\begin{array}{l}\mathrm{i} 2-3.6 \mathrm{~ns} \\
\mathrm{i} 3+2.3 \mathrm{~ns} \\
\mathrm{i} 4-6.7^{\star}\end{array}$ & $+3.6 \%$ & i4 & $\begin{array}{l}\mathrm{i} 1+6.4^{\star} \\
\mathrm{i} 2+5.8 \mathrm{~ns} \\
\mathrm{i} 3+4.8 \mathrm{~ns}\end{array}$ & $+1.4 \%$ \\
\hline $\begin{array}{l}\text { Modèle } 2 \\
\text { Communication } \\
\text { Interne dans les } \\
\text { groupes d'élèves }\end{array}$ & $c 5$ & $\begin{array}{l}\mathrm{cl}-1.7 \mathrm{~ns} \\
\mathrm{c} 2+3.7 \mathrm{~ns} \\
\mathrm{c} 3+3.6 \mathrm{~ns} \\
\mathrm{c} 4-1.9 \mathrm{~ns}\end{array}$ & $+2.4 \%$ & $\mathrm{cl}$ & $\begin{array}{l}\mathrm{c} 2-1.2 \mathrm{~ns} \\
\mathrm{c} 3-2.6 \mathrm{~ns} \\
\mathrm{c} 4+1.1 \mathrm{~ns} \\
\mathrm{c} 5-9.8 \text { * }\end{array}$ & $+4.5 \%$ \\
\hline $\begin{array}{l}\text { Modèle } 3 \\
\text { Profil interactionnel } \\
\text { de la classe } 3\end{array}$ & intl & $\begin{array}{l}\text { Int2 }-0.32 \mathrm{~ns} \\
\text { Int3 }-1.2 \mathrm{~ns} \\
\operatorname{lnt} 4-6.6^{\star} \\
\text { Intinc }+3.0 \mathrm{~ns}\end{array}$ & $+2.5 \%$ & intl & $\begin{array}{l}\text { Int2 }-2.1 \mathrm{~ns} \\
\ln 3+0.8 \mathrm{~ns} \\
\ln 4-7.2^{\star} \\
\text { Intinc }-7.9^{\star}\end{array}$ & $+4.6 \%$ \\
\hline
\end{tabular}

Tableau 4. Récapitulatif de l'effet des pratiques enseignantes sur la progression des élèves pour les deux aptitudes métalinguistiques concernées

Du point de vue de l'efficacité de ces typologies à expliquer les écarts d'acquisition entre les élèves, on observe que c'est la typologie « Implication des élèves » qui contribue le plus à expliquer la variété d'acquisitions de type DECOMP pour une part qui reste cependant assez marginale $(3.6 \%$ contre $16 \%$ pour le niveau initial et $10 \%$ pour les caractéristiques des élèves, $\mathrm{cf}$ tableau 2). Pour MEMDISC ce sont les deux typologies « Profil interactionnel de la classe» et «Communication

Indications pour la lecture du tableau. Exemple pour le modèle 1 : la modalité de la typologie mise en référence est la catégorie 1 (il, a priori la plus favorable aux acquisitions en référence à nos hypothèses), les catégories i2 et i3 présentent un impact non significatif sur les acquisitions des élèves en DECOMP par rapport à il. En revanche la catégorie 4 (i4 a priori la plus défavorable) présente un impact négatif de $6 . \%$ points, significatif au seuil de $10 \%$, par rapport à la catégorie 1. La significativité des coefficients est codée comme suit : ns : non significatif, * significatif au seuil de $10 \%$, ${ }^{\star \star}$ significatif au seuil de $5 \%,{ }^{\star \star \star}$ significatif au seuil de $1 \%$.

2. Gain de variance expliquée par rapport au modèle incluant seulement le niveau initial et les caractéristiques individuelles des élèves.

2. Compte tenu de l'impossibilité de classer les classes 10 et 11 dans cette typologie, elles ont été regroupées dans une catégorie « inconnu » pour ce modèle. interne dans les groupes d'élèves » qui sont les plus « explicatives » (respectivement 4.6 et $4.5 \%$, taux supérieurs au poids du niveau initial : $2.5 \%$, cf. tableau 2). Pour les tâches de décomposition/recomposition d'énoncés (DECOMP) la prise en compte de ces dimensions pédagogiques explique donc au minimum environ un tiers du poids des classes $(2.5 \%$ de variance dans le modèle 3 sur les 
$8 \%$ expliqués par les classes, cf tableau 2), pour les compétences en mémorisation et discrimination auditives un treizième $(1.4 \%$ de variance dans le modèle 1 sur les $18 \%$ expliqués par les classes). C'est dire combien ces dimensions pédagogiques prises en compte lors de l'observation des classes, n'épuisent pas, à elles seules, l'effet-classe observé par ailleurs
- Pour plus de précisions sur les différentes dimensions qui peuvent composer l'effet- classe et leur poids respectif, on peut se rééérer par exemple à DURU-BELLAT M et MINGAT A (1994), « La vaniété de fonctionnement de l'école : identification et analyse des « effets-maitre », in $M$. Crahay, Evaluation et analyse des établissements de formation, De Boeck, Bruxelles.

Si nous examinons à présent plus précisément l'impact des typologies de pratiques d'enseignement-apprentissage sur les progressions des élèves, nous observons tout d'abord qu 'un seul cas ne présente pas d'impact significatif : il s'agit de la typologie «Communication interne dans les groupes d'élèves » pour DECOMP. Dans les autres cas, les résultats vont dans le sens attendu : soit les modalités posées comme les plus favorables aux acquisitions présentent un impact significativement positif par rapport à la modalité défavorable (ex : typologie « Implication » pour MEMDISC), soit la modalité posée comme la plus défavorable aux acquisitions (catégorie 4 ou 5) présente un impact significativement négatif par rapport à la modalité la plus favorable (ex : typologie «Implication » pour DECOMP, typologie « Communication interne » pour MEMDISC, typologie « Interaction » pour les deux aptitudes). L'impact respectif de ces modalités varie de -9.8 points à +6.4 .

Globalement, les hypothèses avancées quant à l'efficacité supposée de certains contextes pédagogiques plus favorables aux acquisitions que d'autres sont donc confirmées par ces résultats. On peut dire notamment, que les classes dans lesquelles l'implication des élèves est forte et celles dans lesquelles la construction des contenus d'apprentissage à travers les échanges entre maîtres et élèves est très interactive constituent des contextes favorables à la progression des élèves dans les deux aptitudes métalinguistiques considérées ici.

On notera toutefois que les modalités intermédiaires des typologie des classes (i2 et i3, c2,c3 et c4, int 2 et int3) ne présentent, le plus souvent, pas d'effet significatif sur la progression des élèves dans les deux compétences. Ce résultat n'est pas inintéressant au plan méthodologique car il signifie que nous ne sommes pas en présence d'un « continuum » de l'efficacité des pratiques observées (qui s'exprimerait grossièrement en disant : plus il y a d'indicateurs « positifs », plus c'est efficace), mais plutôt d'une dichotomie entre des classes ayant des pratiques très opposées et une efficacité également très différenciée. Ce résultat renvoie d'une façon générale à la difficulté d'élaboration de toute typologie et notamment à la question des limites entre catégories. En effet, ces catégories sont fixées de façon relative et sont tributaires du contexte des classes en présence (si on disposait de 10 fois plus de classes, certaines de celles de l'échantillon actuel ne se retrouveraient sans doute pas intégrées dans la même catégorie). La mise en relation avec l'analyse de l'efficacité des classes rend plus lisible cette limite de l'exercice de typologisation : les catégories intermédiaires ne présentant pas d'effet significatif, on peut douter de l'homogénéité des pratiques qu'elles sont censées recouvrir. 


\section{Discussion, perspectives ...}

Au delà des résultats factuels obtenus par cette recherche, sa contribution principale est essentiellement, selon nous, d'ordre méthodologique et ce, sous divers aspects.

Tout d'abord il nous semble que ce travail confirme (comme d'autres l'ont montré précédemment) qu'il est possible de construire des typologies de pratiques à propos de plusieurs dimensions pédagogiques. Plus exactement, il est possible d'objectiver des différences de pratiques d'enseignement-apprentissage selon des critères précis, notamment en ayant recours à une observation approfondie du fonctionnement des classes (même s'il faut bien reconnaître que les moyens mobilisés sont assez « lourds » à mettre en œuvre).

De plus il nous semble établi ici, que ces typologies d'enseignement-apprentissage arrivent à expliquer une partie « des effets classes » (même si cette part reste modeste) et qu'elles « fonctionnent » dans le sens attendu de nos hypothèses. Autrement dit la méthodologie adoptée ici permet de décrire et de mesurer les pratiques qui sont efficaces et celles qui ne le sont pas. En ce sens on peut dire que nos résultats confortent, en quelque sorte, les dimensions de la relation pédagogique que nous avons choisi d'objectiver : degré d'implication des élèves, profil interactionnel des classes et communication interne dans les groupes d'élèves (même si cette dernière dimension semble un peu moins pertinente que les deux autres pour expliquer les différences d'acquisitions entre les élèves ${ }^{\star}$.

- II n'est pas impossible que cette moindre pertinence soit, au moins en partie, due aux pondérations effectuées au plan des indicateurs retenus dans notre grille d'observation des modalités de cette communication interne.

Cependant il nous faut pointer au moins deux limites de notre démarche. Premièrement, si nous avons le sentiment que notre étude peut contribuer à mieux saisir la complexité des actes d'enseignement/apprentissage et, conjointement, à isoler quelques variables émergentes • Voir à ce sujet de Pietro et al. (2003) " porteuses d'efficacité » ainsi qu'à mettre à jour quelques configurations plus «favorables » aux acquisitions des élèves, il va de soi que ces dimensions ne sont certainement pas « modélisables » au sens d'une quelconque prescription. La prudence est de mise car « le même comportement peut avoir des effets inverses selon le contexte interactif dans lequel il se déroule » (Felouzis, 1997) On ne peut notamment pas considérer l'acte d'enseignement comme une somme de pratiques plus ou moins favorables aux acquisitions des élèves.

- Voir également Bressoux, 1990 et 1994 à ce sujet.

De ce point de vue, nous avons au cours de ce travail, testé un modèle dans lequel nous avons opposé les classes « bien placées » dans les trois typologies (classes 7,9 et 12) aux trois autres «mal placées » (classes 2, 3 et 16). Il s'avère que le premier groupe ne présente pas d'efficacité significative par rapport au second dans aucun des deux domaines d'acquisitions évaluées. Ce résultat confirme donc qu'il ne suffit pas que tous les indicateurs des dimensions pédagogiques soient « au vert » pour que l'efficacité maximale soit atteinte. Ceci tient sans doute au fait que l'acte d'enseigner représente une sorte de résultante de contraintes multiples et souvent contradictoires, liées à un contexte donné, et que chaque enseignant 
peut résoudre cette complexité de façon différente. C'est pour cette raison que nous rencontrons des difficultés à expliquer pourquoi, dans certaines classes, les élèves apprennent tout de même malgré la faiblesse des indicateurs pédagogiques retenus et pourquoi dans certaines autres les élèves n'apprennent guère quand bien même tout s'y passe plutôt favorablement (du point de vue de nos hypothèses) au plan des pratiques...

Par ailleurs, le travail exploratoire présenté ici met en évidence, ainsi que nous l'avons déjà signalé plus haut, les limites des approches typologiques. Celles-ci sont dues d'une part aux difficultés d'établir des frontières autres qu'arbitraires entre les catégories, d'autre part à la difficulté de construction même des indicateurs qui en sont la baset.

- Voir à ce sujet I'article de S.Aeby et J.F De Pietro dans ce numéro, à propos de la variable SOCIO notamment.

Pour conclure, le travail présenté ici constitue la dernière phase d'un triple processus. Il porte l'empreinte des transformations de la sociologie de l'éducation française durant les trois dernières décennies (du système à l'acteur); il est le reflet d'un travail d'équipe qui permet d'adjoindre différents regards scientifiques (et les méthodologies afférentes : analyses multivariées, observations écologiques de classes, apports des analyses conversationnelles...), et enfin, il traduit un cheminement ayant conduit à revisiter le paradigme "processus-produit" par étapes successives (Genelot, 2001 ; Tupin, 2001, Genelot et Tupin, 2001 ; De Pietro, Aeby, Genelot et Tupin, 2003) ménageant l'évolution d'une évaluation externe à un regard interne qui réhabilite notamment le rôle de l'élève dans la (co-)construction de ses connaissances. En bref, nos recherches, initialement plus proches du versant "enseignement" (et de ses effets) se sont progressivement rapprochées d'une conception plus large intégrant la dimension interactive du processus "d'enseignement-apprentissage" (CREN, 1999).

A ce titre, les résultats obtenus renforcent notre conviction de l'intérêt qu'il y a à connecter des approches qualitatives d'observation des pratiques de classes avec des approches quantitatives d'évaluation de l'efficacité. Toutefois, pour dépasser les limites mentionnées plus haut, il faudrait notamment parvenir à travailler avec des modèles qui expliqueraient directement l'efficacité des classes (variable dépendante) par un certain nombre de dimensions pédagogiques (dont on pourrait comparer l'efficacité entre elles). Une telle démarche nécessite, cependant, un échantillon de classes de grande taille peu compatible avec un dispositif d'observation «lourd» comme c'était le cas ici. L'enjeu serait donc de rechercher des indicateurs pédagogiques à la fois relativement « faciles » à observer et discriminants au plan de l'efficacité. Dans notre recherche, il nous semble que l'outil « implication des élèves » répond davantage à cette exigence que l'outil « interaction », par exemple. On ne peut donc que souhaiter que les nombreux travaux d'observation des classes actuellement engagés par diverses équipes de recherche, permettent dans les années qui viennent de décrire des observables simples mais discriminants pouvant être intégrés ensuite dans des approches quantitatives. 


\section{Bibliographie}

ALTET, M. (1993). Styles

d'enseignement, styles pédagogiques, in $\mathrm{J}$.

Houssaye, La pédagogie : une encyclopédie pour aujourd'hui, Paris : ESF

ALTET, M. BRESSOUX, P. BRU, M. LECONTE-LAMBERT, C. (1994) et (1996a). Étude exploratoire des pratiques d'enseignement en classe de CE2. Les dossiers d'Education et Formations. MEN, Direction de l'évaluation et de la prospective, $n^{\circ} 44$ et 70 .

ALTET, M. BRESSOUX, P. BRU, M.

LECONTE-LAMBERT, C. (1996b). Les caractéristiques des pratiques d'enseignement au CE2 : vers une meilleure connaissance de l'effet-maître. Education et formations, 46, 71-80 BRESSOUX, P. (1990). Méthodes pédagogiques et interactions verbales dans la classe : quel impact sur les élèves de CP ?, Revue française de pédagogie, $\mathrm{n}^{\circ} 93$.

BRESSOUX, P. (1994). Les recherches sur les effets-écoles et les effets-maîtres, Revue Française de Pédagogie, ${ }^{\circ} 108$, 91-137

BRESSOUX, P., BRU, M., ALTET, M., LECONTE-LAMBERT, C. (1999).

Diversité des pratiques d'enseignement à l'école élémentaire, Revue française de pédagogie, $\mathrm{n}^{\circ} 126,97-110$.

BRESSOUX, P. (2000). Modélisation et évaluation des environnements et des pratiques d'enseignement, Rapport d'Habilitation à Diriger des Recherches, Université Pierre Mendes France

BRESSOUX, P. (2001). Réflexions sur l'effet-maître et l'étude des pratiques enseignantes. Les Dossiers des sciences de l'Education, $\mathrm{n}^{\circ}$ 5, Toulouse : Presses Universitaires du Mirail, 35-52.

BRU, M. (1992). Les variations didactiques dans l'organisation des conditions d'apprentissage, Toulouse : Editions universitaires du Sud.

BRU, M. (coord.) (2001). Les pratiques enseignantes : contributions plurielles, Les Dossiers des sciences de l'Education, $\mathrm{n}^{\circ} 5$, Toulouse : Presses Universitaires du Mirail.
BRU, M. (2002). Pratiques enseignantes : des recherches à conforter et à développer. Revue Française de Pédagogie, $\mathrm{n}^{\circ} 138$.

CANDELIER, M., (1998). L'éveil aux langues à l'école primaire, le programme européen EVLANG, De la didactique des langues à la didactique du plurilinguisme, Hommage à Louise Dabène, Grenoble, CDL- Lidilem, 299-308

CANDELIER, M. (dir.) (2003). EVLANG : l'éveil aux langues à l'école primaire. Bilan d'une innovation. Bruxelles : De Boeck.(à paraître). CLANET, J. (1997). Contribution à l'intelligibilité du système denseignementapprentissage. Stabilisations du système et interactions en contexte. Thèse de Doctorat Nouveau Régime, sous la dir. de M. Bru, Université de Toulouse-Le Mirail CREN (1999) Analyse plurielle d'une séquence d'enseignement-apprentissage, Les cahiers du CREN, CRDP, Nantes DE PIETRO, J-F.,AEBY, S., GENELOT,S., TUPIN, F.(2003). Des interactions aux apprentissages : vers une mise en évidence de "l'effet classe». Colloque « Construction des connaissances et langage dans les disciplines d'enseignement. DAEST, EA 256. Université de Bordeaux II, IUFM Aquitaine. Bordeaux, 3-5 avril 2003. DELHAXHE A. (1997), Le temps comme unité d'analyse dans la recherche sur l'enseignement. Revue Française de pédagogie, $\mathrm{n}^{\circ} 118$.

DURU-BELLAT, M. ET MINGAT, A. (1994). La variété de fonctionnement de l'école : identification et analyse des « effets-maître », in M. Crahay, Evaluation et analyse des établissements de formation, De Boeck, Bruxelles. FELOUZIS,G., (1997). L'efficacité des enseignants, Paris : PUF.

GENELOT, S. (2001). Evaluation qualitative du curriculum "Evlang ", Rapport de recherche auprès de la Commission européenne, Projet Socrates/Lingua 42137-CP-2-981-FRLingua-LD, «Eveil aux langues », Bruxelles. 
GENELOT, S. ET TUPIN, F., (2001).

Evaluation des pratiques pédagogiques : les atouts d'une approche méthodologique plurielle. Le cas du programme EVLANG. Communication au 4ème congrès de l'AECSE, Lille, 5-8 sept. 2001. (CD ROM)

GENELOT, S. ET TUPIN, F., (2003). Méthodologie de l'évaluation, in EVLANG-L'éveil aux langues à l'école primaire. Bilan d'une innovation européenne. (sous la dir. de M. Candelier) Chapitre 4, Bruxelles : De Boeck Université. (à paraître)

KERBRAT-ORRECHIONI, C. (1990). Les interactions verbales. Paris, A. Colin. (3 tomes)

MINGAT, A. (1984). Les acquisitions scolaires des élèves au $\mathrm{CP}$ : les origines des différences ?, Revue Française de Pédagogie, ${ }^{\circ} 69$.
ROULET, E. (1985). L'articulation du discours en français contemporain, Berne, Peter Lang.

SUCHAUT B (1996). La gestion du temps à l'école maternelle et primaire : diversité des pratiques et effets sur les acquisitions des élèves, L'année de la recherche en éducation, 123-153.

TUPIN F., (2001), (dir.), Evaluation qualitative des processus et effets liés au curriculum «Evlang », Rapport de recherche auprès de la Commission Européenne, Projet Socrates/Lingua 42137-CP-2-981-FR-Lingua-LD, « Eveil aux langues », Bruxelles. 